\title{
Melanoma: un desafío para el pediatra. Dos casos clínicos
}

\author{
Melanoma: A pediatric challenge. A two cases report
}

Dra. Tatiana Bendersky, ${ }^{a}$ Dra. Daniela Berlingieri, ${ }^{a}$ Dra. Sonia Rojas ${ }^{a}$ y Dr. Ignacio Nuñez ${ }^{a}$

\section{RESUMEN}

El melanoma es la forma más grave de cáncer de piel. La morbimortalidad es variable, ya que se relaciona con las medidas de prevención implementadas, la detección temprana y el acceso al tratamiento temprano. La incidencia ha aumentado en los últimos años a pesar de la mayor concientización con respecto a la exposición a la luz solar y la utilización de cremas protectoras. A su vez, el rango etario se ha ampliado, y esta enfermedad afecta a individuos cada vez másjóvenes. Se estima que del $1 \%$ al $4 \%$ de todos los casos de melanoma ocurren en menores de 20 años.

Se presentan dos casos pediátricos, con evolución prolongada y diagnóstico inicial erróneo. Es fundamental para el pediatra general, primer contacto del paciente con el sistema de salud, conocer las características de estas lesiones. Un alto índice de sospecha permitiría la derivación al especialista de forma temprana.

Palabras clave: melanoma, pediatría, diagnóstico.

\begin{abstract}
Melanoma is the most serious skin cancer. Morbimortality is variable as it is related to the preventive measures, early detection, and access to early treatment. The incidence has increased in recent years, despite the raise in awareness of avoiding sun exposure and the use of sunscreen. What is more, the disease age range has expanded, affecting increasingly younger individuals. It is estimated that 1 to $4 \%$ of all melanoma cases occurred in people younger than twenty years old.

We present two pediatric cases with prolonged evolution and wrong initial diagnosis. Since the general pediatrician is the patient's first contact with the health system, it is extremely important to know the characteristics of these lesions. A high index of suspicion would allow an early referral to the specialist. Key words: melanoma, pediatrics, diagnosis.
\end{abstract}

http: / / dx.doi.org/10.5546/ aap.2021.e536

Cómo citar: Bendersky T, Berlingieri D, Rojas S, Nuñez I. Melanoma: un desafío para el pediatra. Dos casos clínicos. Arch Argent Pediatr 2021;119(5):e536-e539.

a. Clínica Pediátrica. Hospital de Niños Ricardo Gutiérrez, Ciudad Autónoma de Buenos Aires, Argentina.

Correspondencia:

Dra. Tatiana Bendersky: tatianabendersky@gmail.com

Financiamiento: Ninguno.

Conflicto de intereses: Ninguno que declarar.

Recibido: 22-12-2020

Aceptado: 30-3-2021

\section{INTRODUCCIÓN}

El término melanoma se refiere a aquella neoplasia maligna que proviene de las células melanocíticas de la piel. Estas células también están presentes en los folículos pilosos, las leptomeninges, el oído interno y la coroides del ojo.

A pesar de que en pediatría continúa siendo infrecuente, durante el período 2000-2016 se notificaron en Argentina 80 casos nuevos de melanoma en niños, de los cuales el $55 \%$ ocurrió entre los 10 y los 14 años, con un promedio de 4,7 casos/año. ${ }^{1}$

Debido a la baja incidencia en esta población, quedan aspectos sin definir en relación a la presentación clínica o al comportamiento biológico de estos tumores, siendo frecuente que se presente como una lesión inespecífica, no pigmentada o de aspecto benigno que lleva a realizar un diagnóstico erróneo inicial, tratamiento tardío y por lo tanto peor pronóstico. ${ }^{2}$

\section{Caso clínico 1}

Paciente de 3 años, sin antecedentes personales ni familiares, es llevado a la consulta con el pediatra por presentar lesión cutánea de un año y medio de evolución en el antebrazo derecho, amelanótica, no dolorosa ni pruriginosa, de $0,4 \times 0,6 \mathrm{~cm}$. Se indicó sulfadiazina argéntica local sin evidenciar mejoría clínica (Figura 1). Se derivó al Servicio de Dermatología, donde se realizó una biopsia de la lesión. La anatomía patológica informó “Melanoma nodular. Márgenes de resección laterales libres de melanoma, focalmente comprometidos por nevo displásico. Margen profundo libre. Clark IV. Breslow 2,2 mm".

Se derivó a la niña a un centro de mayor complejidad para evaluación. Al ingreso presentaba, en el antebrazo derecho, una cicatriz en la región biopsiada, sin otra signosintomatología para destacar. El laboratorio general y la tomografía computada con emisión de positrones (PET-TC, por su sigla en inglés) no informaron hallazgos relevantes. Se realizó marcación y resección del ganglio centinela axilar y epitroclear, ninguno mostró infiltración. La ampliación de márgenes de la lesión no evidenció enfermedad maligna. Por no presentar 
enfermedad diseminada, la resección previa se consideró curativa y no realizó tratamiento sistémico.

Continuó el seguimiento en los servicios de Oncología y Dermatología con controles trimestrales con PET-TC. Luego de doce meses, presenta una evolución favorable, con últimos estudios sin evidencia de enfermedad.

\section{Caso clínico 2}

Paciente de 9 años, sin antecedentes personales o familiares de relevancia, llevado a la consulta por presentar en el brazo izquierdo una lesión violácea, sobreelevada, de $0,7 \times 0,5 \mathrm{~cm}$, de consistencia duro-elástica, superficie escoriada, no dolorosa ni pruriginosa, de un año de evolución (Figura 2). Se diagnosticó molusco contagioso y recibió tratamiento tópico con distintas cremas, sin observar mejora y con progresión del tamaño y la consistencia de la lesión. Realizó una consulta en el Servicio de Dermatología, donde se indica biopsia de la lesión. La anatomía patológica informó "Melanoma epitelioide con márgenes de resección que pasan por la lesión, Clark V. Inmunohistoquímica de la lesión positiva para S100, Bcl-2, ciclina D1 y p53". Se realizó PET-

Figura 1. Lesión sobreelevada, amelanótica, de 0,4 $\times 0,6 \mathrm{~cm}$ con bordes eritematosos en antebrazo derecho (paciente del caso 1)

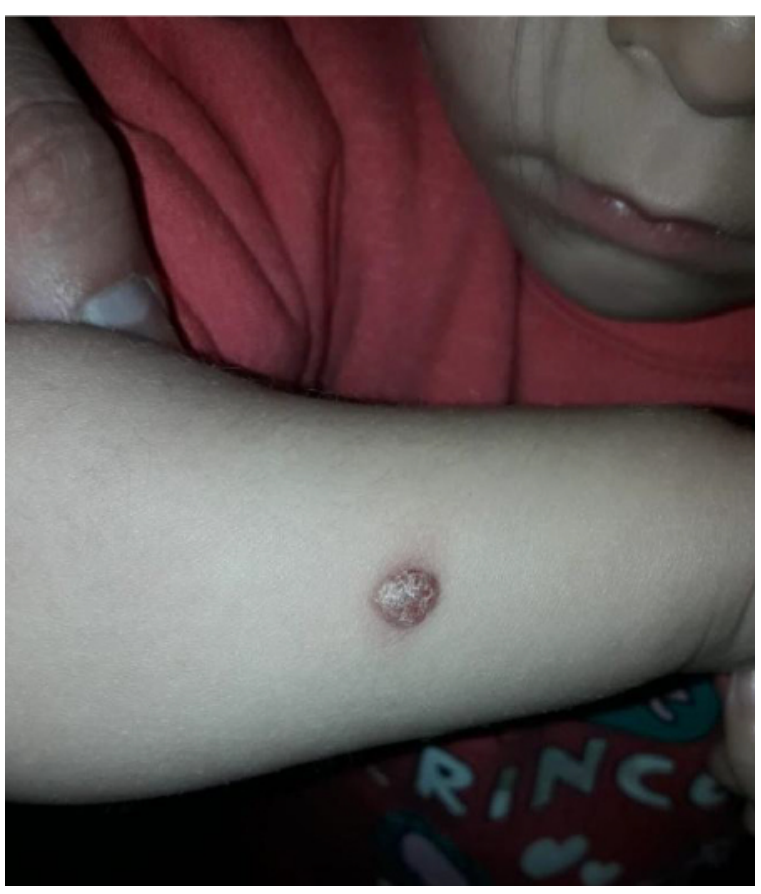

TC, con resultado normal, y se internó para completar la evaluación. Al ingreso, el paciente no presentaba hallazgos patológicos al examen físico, solo la lesión cicatricial posbiopsia. Presentaba laboratorio general dentro de límites normales. Se realizó la ampliación de márgenes de resección de la lesión y la marcación del ganglio centinela axilar izquierdo. La nueva biopsia informó melanoma epitelioide (Clark V Breslow $7 \mathrm{~mm}$ ) con márgenes laterales libres de lesión. De los dos ganglios centinelas marcados, uno presentaba infiltración metastásica. Por enfermedad diseminada, inició tratamiento sistémico con pembrolizumab.

Luego de doce meses de seguimiento, continúa con este tratamiento y se realizan PETTC seriadas cada 3 meses, las cuales se mantienen con resultados normales.

\section{DISCUSIÓN}

Los factores de riesgo para padecer melanoma son la alta exposición a la luz solar, el antecedente de quemaduras solares, presentar fototipo cutáneo I y II, tener pecas, historia de melanoma en familiares de primer grado y patologías que afectan la reparación del ADN como es la

FIgURA 2. Lesión sobreelevada de 0,7 $\mathrm{mm} \times 0,5 \mathrm{~mm}$, de consistencia duro-elástica, violácea, con superficie excoriada en el brazo izquierdo (paciente del caso 2)

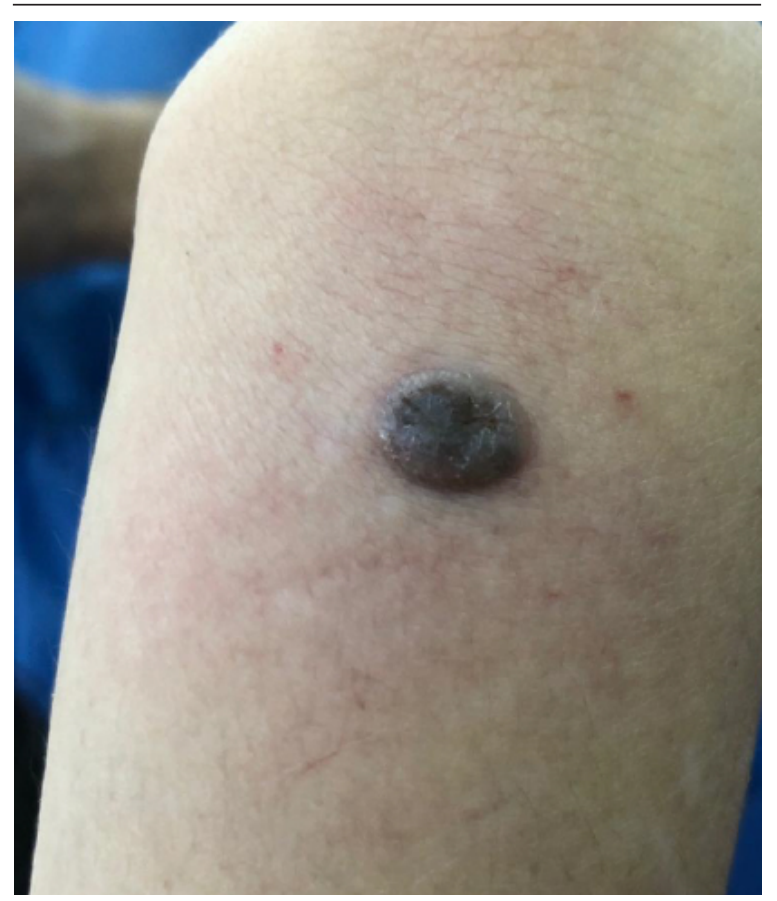


xerodermia pigmentosa (Tabla 1).3,4 A pesar de que muchos melanomas surgen de nevos congénitos, en los pacientes pediátricos la mayoría surgen de novo. ${ }^{5,6}$

Uno de los factores evitables es la exposición a la luz solar. El $80 \%$ del daño que genera se produce en los primeros 18 años de vida, y es acumulativo. ${ }^{2,6} \mathrm{~A}$ su vez, la piel en este grupo etario es más fotosensible, y los factores de riesgo de desarrollar melanoma ya se encuentran presentes. ${ }^{4}$ Esto explica que en la adolescencia el lugar de aparición del melanoma es más frecuente en zonas de alta exposición, mientras que en la infancia se manifiesta en zonas expuestas y no expuestas por igual, ${ }^{7}$ principalmente en cabeza y cuello. ${ }^{5,6}$

En el 2009, la Organización Mundial de la Salud clasificó a la exposición a los rayos ultravioletas (UV) como un carcinógeno de grado I. ${ }^{8}$ Aquí radica la importancia de las recomendaciones de la Sociedad Americana de Pediatría, que indica evitar la exposición a la luz solar al mediodía, usar ropa adecuada que proteja del sol, lentes con protección UV, sombrero que proteja la cara, y usar crema protectora (para rayos UVB y UVA, con un factor de protección mínimo de 15) y reponerla cada 2 horas. Se recomienda no exponer al sol a los menores de 6 meses. ${ }^{8,9}$

La presentación de la enfermedad difiere según la edad. En los niños, es más frecuente la presentación atípica con lesiones de mayor espesor, sobreelevadas, ulceradas y con más probabilidad de lesiones metastásicas, ya que suelen ser de rápido crecimiento y más agresivos. ${ }^{5,6} \mathrm{El} 80 \%$ de los melanomas son amelanóticos, lesiones rojas o rosadas mientras que, en los adolescentes solo un $23 \%$ de las lesiones tienen estas características. ${ }^{10}$

Estas diferencias en pediatría suelen demorar y confundir el diagnóstico, y constituyen un desafío aun para los especialistas, tal como sucedió con el paciente del caso 1, en quien la forma de presentación fue una lesión amelanótica que, al inicio, no fue sospechosa de malignidad.

El estudio de Cordoro et al., ${ }^{11}$ demostró que la regla de $\mathrm{ABCDE}$ (asimetría, bordes irregulares, variación de color, diámetro $>6$ mm y evolución) utilizada en los pacientes adultos no fue útil en niños. En este estudio retrospectivo, la mayoría de los pacientes pediátricos de entre 0 y 9 años se presentaban con lesiones amelanóticas, bordes regulares, color uniforme, simetría y diámetro de $6 \mathrm{~mm}$ o menos; en los pacientes de entre 10 años y 20 años, la mitad cumplía con la regla.

Estos autores proponen nuevos criterios para la evaluación de lesiones en pediatría siguiendo con el mismo acrónimo, pero con mayor sensibilidad en este grupo etario. Establecen que: $\mathrm{A}=$ amelanosis, $\mathrm{B}=$ lesiones sangrantes, sobreelevadas, $\mathrm{C}=$ color uniforme, $\mathrm{D}=$ de novo con cualquier diámetro. Cabe destacar que la letra E (evolución) también resulta de importancia en el seguimiento de cualquier lesión de piel., ${ }^{9,11}$ Familiarizarse con estos criterios nuevos aceleraría el diagnóstico ya que, de no tenerlos en cuenta, el $82 \%$ se demoraría más de 6 meses, ${ }^{9}$ como sucedió en los casos presentados.

TABLA 1. Factores de riesgo para padecer melanoma

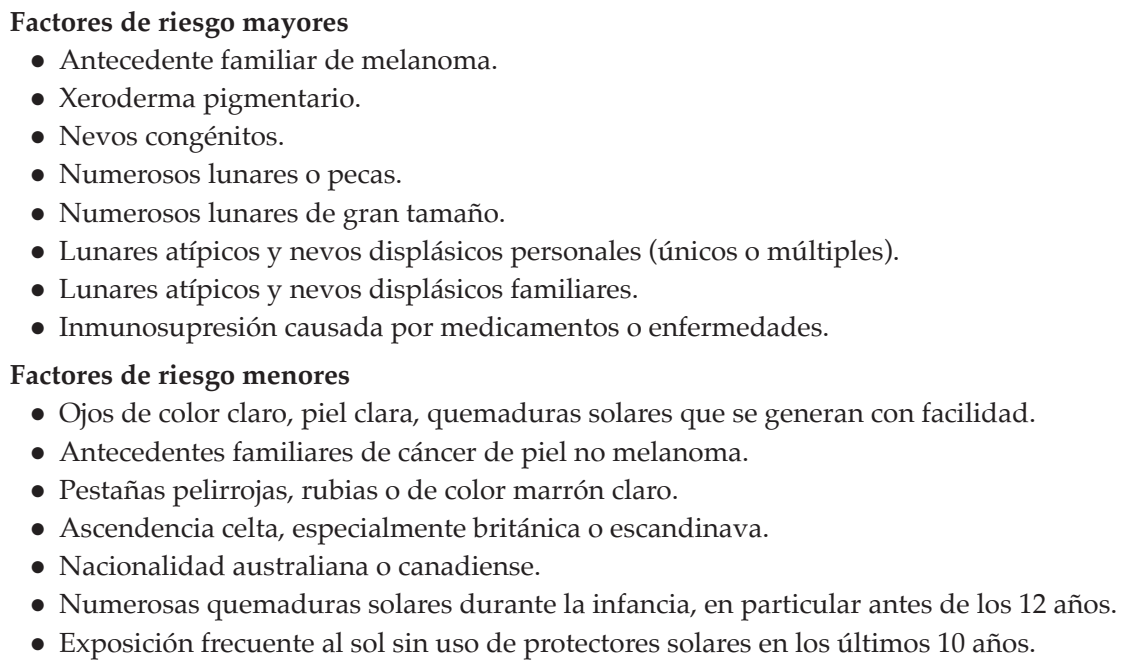


Los diagnósticos diferenciales incluyen nevos de Spitz atípicos, nevos melanocíticos congénitos y adquiridos, nevus spilus, molusco contagioso y el nevo azul. ${ }^{5}$

Una vez que se identifica una lesión sospechosa, la biopsia escisional está indicada. No se recomienda hacer curetaje. ${ }^{9}$ El manejo posterior y el pronóstico están relacionados con el estadio de la enfermedad, determinado por la profundidad de la lesión expresado en el nivel de Clark (nivel de invasión respecto a las estructuras de la piel) y el espesor de Breslow (medida del grosor del melanoma en milímetros), ${ }^{3}$ así como también con la presencia de metástasis.

El manejo quirúrgico de la lesión depende de la estadificación tumoral. En aquellas lesiones menores de $1 \mathrm{~mm}$ ulceradas o con elevado índice mitótico y en aquellas mayores de $1 \mathrm{~mm}$ de espesor, se realiza la biopsia del ganglio centinela $\mathrm{y}$, si resulta positiva, se procede a su exéresis. Aquellos ganglios que se encuentran clínicamente comprometidos son removidos. ${ }^{9}$ Los niños con biopsia de ganglio centinela positivo presentan mayor sobrevida en comparación con los adultos, pero aquellos niños con ganglio centinela positivo presentan peor sobrevida que aquellos que no lo tienen. ${ }^{12}$

El papel diagnóstico de la PET-TC también depende del estadio del melanoma. Para los estadios I y II no se recomienda su realización, ya que presenta tasas elevadas de falsos positivos en detectar metástasis. Sin embargo, para los estadios III y IV, es la imagen con mayor sensibilidad y especificidad para detectarlas. ${ }^{13}$

El tratamiento adyuvante con quimioterapia se extrapola de estudios realizados en adultos. Faltan estudios de investigación destinados a comprobar la eficacia de estos fármacos en pediatría. ${ }^{9}$

Los casos presentados enfatizan que el papel del pediatra es fundamental a la hora de reconocer los factores de riesgo de melanoma, así como también proporcionar a los pacientes las recomendaciones para evitar su aparición. Está comprobado que su identificación, el reconocimiento temprano de las lesiones primitivas, evitar la exposición solar y la fotoprotección desde temprana edad pueden ayudar a reducir la morbilidad y la mortalidad de esta patología.

\section{REFERENCIAS}

1. Moreno F, Chaplin MA. Registro Oncopediátrico Hospitalario Argentino. 6ta ed. Buenos Aires: Instituto Nacional del Cáncer; 2018. [Acceso: 21 de junio 2020]. Disponibleen https: / / bancos.salud.gob.ar/sites/default/ files / 2020-07 / registro-oncopediatrico-argentino-digital. pdf

2. Averbook BJ, Lee SJ, Delman KA, Gow KW, et al. Pediatric melanoma: analysis of an international registry. Cancer. 2013; 119:4012-9.

3. Orlow SJ. Melanomas in Children. Pediatr Rev. 1995; 16(10):365-9.

4. Brodkin RH, Altman EM. Controlling Malignant Melanoma: A Focus on Pediatricians. Am J Dis Child. 1993; 147(8):875-81.

5. Slade AD, Austin MT. Childhood melanoma: an increasingly important health problem in the USA. Curr Opin Pediatr. 2014; 26(3):356-61.

6. Dinulos J. Childhood melanoma: what every pediatrician should know. Curr Opin Pediatr. 2009; 21(4):472-4.

7. Lange JR, Palis BE, Chang DC, Soong SL, Balch CM. Melanoma in children and teenagers: an analysis of patients from the National Cancer Data Base. J Clin Oncol. 2007; 25(11):1363-8.

8. Long M. Sun Exposure. Pediatr Rev. 2017; 38(9):446-7.

9. Saiyed FK, Hamilton EC, Austin MT. Pediatric melanoma: incidence, treatment, and prognosis. Pediatric Health Med Ther. 2017; 8:39-45.

10. Austin MT, Xing Y, Hayes-Jordan AA, Lally KP, Cormier JN. Melanoma incidence rises for children and adolescents: an epidemiologic review of pediatric melanoma in the United States. J Pediatr Surg. 2013; 48(11):2207-13.

11. Cordoro KM, Gupta D, Frieden IJ, McCalmont T, KashaniSabet M. Pediatric melanoma: results of a large cohort study and proposal for modified ABCD detection criteria for children. J Am Acad Dermatol. 2013; 68(6):913-25.

12. Kim J, Sun Z, Gulack BC, Adam MA, et Al. Sentinel lymph node biopsy is a prognostic measure in pediatric melanoma. J Pediatr Surg. 2016; 51(6):986-90.

13. KasteSC.Imaging of pediatric cutaneous melanoma.Pediatr Radiol. 2019; 49(11):1476-87. 